\title{
Core-shell nanoparticles with self-regulating plasmonic functionality
}

\author{
M. B. Cortie*, A. Dowd, N. Harris, and M. J. Ford \\ Institute for Nanoscale Technology, University of Technology Sydney, PO Box 123, Broadway, NSW \\ 2007, Australia
}

\section{(RECEIVED DATE)}

We propose a smart nanoparticle, a 'regulatron', that exploits a cycle of dynamic plasmonic feedback to self-regulate its temperature to a fixed range. One kind of regulatron can be conceived from $\mathrm{VO}_{2}$ and $\mathrm{Au}$; the temperature of this particle when illuminated by laser irradiation of suitable intensities and wavelengths is $\sim 340 \mathrm{~K}$. We expect that regulatrons of this and other compositions will be useful in applications such as photothermal medical therapeutics.

PACS : 64.70.Kb, 71.45.Gm, 78.67.Bf, 81.40.Tv

Morin's discovery[1] in 1959 that vanadium dioxide underwent a metal-insulator transition has stimulated much interest in this material. The semi-conducting, monoclinic form of vanadium dioxide (' $\mathrm{VO}_{2}-\mathrm{M}_{1}{ }^{\prime}$ ) transforms reversibly to a metallic, tetragonal structure (' $\mathrm{VO}_{2}-\mathrm{R}$ ') when heated above $\sim 340 \mathrm{~K}\left(67^{\circ} \mathrm{C}\right)$, a transition attended by large changes in conductivity and optical properties[1-3]. This phenomenon has inspired the development of $\mathrm{VO}_{2}$ as the active component of 'smart' window coatings that reflect sunlight when the glass gets hot, e.g.[4], as well as its use in optical switching or measurement devices, e.g.[5]. However, at least one other intriguing application for this fascinating material seems to have been overlooked. Here we will show that composite $\mathrm{Au}-\mathrm{VO}_{2}$ nanoparticles of specific dimensions will regulate temperature through a process of dynamic feedback during illumination by a laser. We name this device a 'regulatron'. An optically-driven nanoscale temperature regulator will have far-reaching impact, an obvious example of which will be to prevent 'run-away' heating[6] in photothermal medical therapeutics[7-9]. The conceptual $\mathrm{Au} / \mathrm{VO}_{2}$ 'regulatron' to be described here is the prototype for this previously unforeseen class of mesoscale plasmonic devices, and 
we believe that it will be followed by regulatrons based on other materials, such as $\mathrm{SmS}$, NiS and Ga, that also have phase transitions accompanied by a large changes in optical properties.

The starting point for constructing these devices are precious metal nanoparticles, which can absorb anomalously large amounts of light relative to their geometric cross-section across a relatively small wavelength region. This absorption arises through excitation of plasmon resonances on the particle, [10]. For gold spheres in vacuum and with diameters of 10 to $80 \mathrm{~nm}$ the resonance occurs at approximately $520 \mathrm{~nm}$. The overall extinction coefficient of the particle is the sum of light absorbed and light scattered, but it is only the absorbed portion that interests us here. This is converted to thermal energy, causing the phenomenon of 'plasmonic heating' $[6,11]$. Some discussion of the exploitation of plasmonic heating in nanoparticles may be found elsewhere[7, 9, 11-13] and it is sufficient to note here that an ability to modulate this effect would be of potential interest in both solar glazing and photothermal therapeutics, to name only two possibilities. For metals such as gold and silver the resonance of a nanosphere (and other particle shapes) is red-shifted when the refractive index of the surrounding medium is increased. Several methods to produce nanoparticles of complex shape or structure have recently been developed[14] and the effect of changes in the dielectric properties of the medium are amplified with some of the new shapes[15]. Nanoshells of gold (on a dielectric core) or nanorods of gold or silver with varying aspect ratio provide a potent means to tune the plasmon resonance from the visible to near infra-red[14].

The combination of the two effects described above, metal-insulator transition and plasmonic absorption, opens a range of new possibilities for creating composite meta-materials with optical properties that are strongly temperature dependent. This possibility has been but sparsely explored, with only a few reports appearing regarding the effect of temperature on the plasmon resonance of $\mathrm{Ag}$ or $\mathrm{Au}$ nanospheres embedded in a $\mathrm{VO}_{2}$ matrix[16-18], of composite $\mathrm{Au}-\mathrm{VO}_{2}$ films[19] or of $\mathrm{VO}_{2} @ \mathrm{Au}(\mathrm{Au}-$ on- $\mathrm{VO}_{2}$ ) core-shell particles[20]. Curiously, however, the possibility of self-regulating temperature control in these structures appears to have been hitherto overlooked.

Here we examine, using computational methods, how the optical properties of various forms of composite nanoparticles of $\mathrm{Au}$ and $\mathrm{VO}_{2}$ would be affected by the phase transition in $\mathrm{VO}_{2}$, and from this field select a particular particle geometry for a more detailed analysis. Particles of spherical symmetry were modeled using the 'BHCOAT' code of Bohren and Huffman[6, 10], while a less symmetrical nanorod shape was modeled with the discrete dipole approximation of Draine and Flatau[21]. Although our assertions are at this stage based only on the results of calculations, the excellent predictive capabilities[10, 14] of the codes used gives us confidence in the results. The dielectric properties of $\mathrm{VO}_{2}-\mathrm{M}_{1}$ and $\mathrm{VO}_{2}-\mathrm{R}$ were taken from Verleur et al.[22] and were originally determined at $300 \mathrm{~K}$ and 
$355 \mathrm{~K}$ respectively. In the present work we have ignored any small variations of optical properties with temperature in the range 300 to $400 \mathrm{~K}$, other than those induced by the phase transition itself, a simplification which we assert does not compromise the generality of our conclusions.

To explain the 'regulatron' effect we consider first a simple Au nanosphere of $40 \mathrm{~nm}$ diameter that is coated with a $10 \mathrm{~nm}$ thick shell of $\mathrm{VO}_{2}$ (a configuration designated $\mathrm{Au} @ \mathrm{VO}_{2}$ ), with the composite particle surrounded by vacuum. The presence of the $\mathrm{VO}_{2}$ shell causes the plasmon resonance to red-shift by $\sim 70 \mathrm{~nm}$ relative to the naked gold sphere, but if the temperature of the particle is raised above that of the $\mathrm{M}_{1} \rightarrow \mathrm{R}$ phase transformation, there will be a sharp change in the refractive index of the $\mathrm{VO}_{2}$, and the peak of the plasmon resonance moves back in the blue direction by $\sim 20 \mathrm{~nm}$. The effect will be reversible, subject only to the small hysteresis in transformation temperatures expected for the transition. This phenomenon is in itself not new having already been demonstrated for Au nanoparticles in a continuous matrix of $\mathrm{VO}_{2}[16-18]$, e.g. Figure 1a.

The effect is obviously magnified for a greater difference in the optical properties of the high and low temperature forms of the structure. The maximum possible values of the ratio,

$$
\phi=\frac{Q_{a b s}^{V O_{2}-M_{1}}}{Q_{a b s}^{V O_{2}-R}}
$$

for a range of $\mathrm{Au} @ \mathrm{VO}_{2}$ particles taken over wavelengths in the interval 300 to $1800 \mathrm{~nm}$ are shown in Figure 1b. (Although this optimization could also have been performed in respect of $Q_{\text {ext }}$, it is the magnitude of $Q_{\mathrm{abs}}$ that controls plasmonic heating and hence of interest here.) The optimum value of $\phi=2.56$ is achieved for these particles when the core is $58 \mathrm{~nm}$ in diameter and the $\mathrm{VO}_{2}$ shell has a thickness of $16.5 \mathrm{~nm}$, and this occurs at $\lambda=680 \mathrm{~nm}$, Figure 1c, which represents the optimum geometry for $\mathrm{Au} @ \mathrm{VO}_{2}$ particles. A similar effect is achievable if $\mathrm{VO}_{2}$ sheaths or end caps are overcoated on a gold nanorod, Figure 1d. We have not attempted to optimize this effect in this case, and the purpose in showing it here is merely to demonstrate that it will also be feasible to use nanoparticles of other than spherical shape to achieve a differential optical absorption. It is obviously also possible to apply a $\mathrm{VO}_{2}$ outer shell on top of an existing Au nanoshell, to give for example a $\mathrm{SiO}_{2} @ \mathrm{Au} @ \mathrm{VO}_{2}$ particle. 




(a)

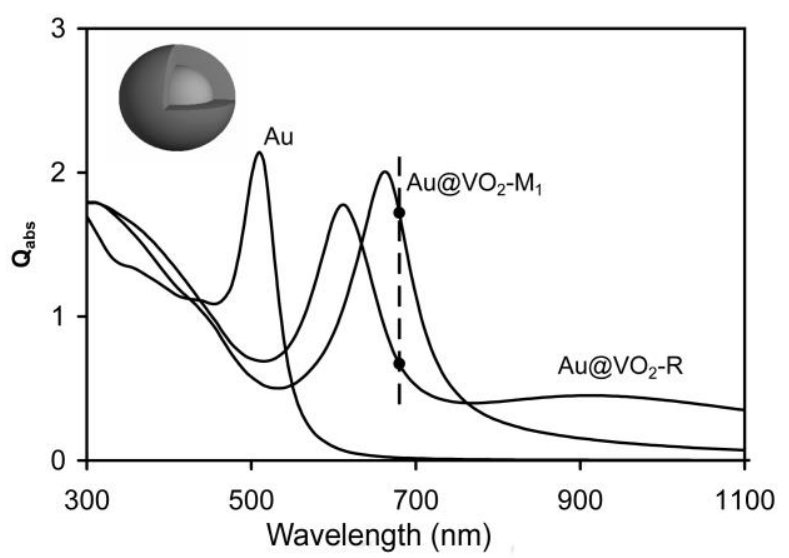

(c)

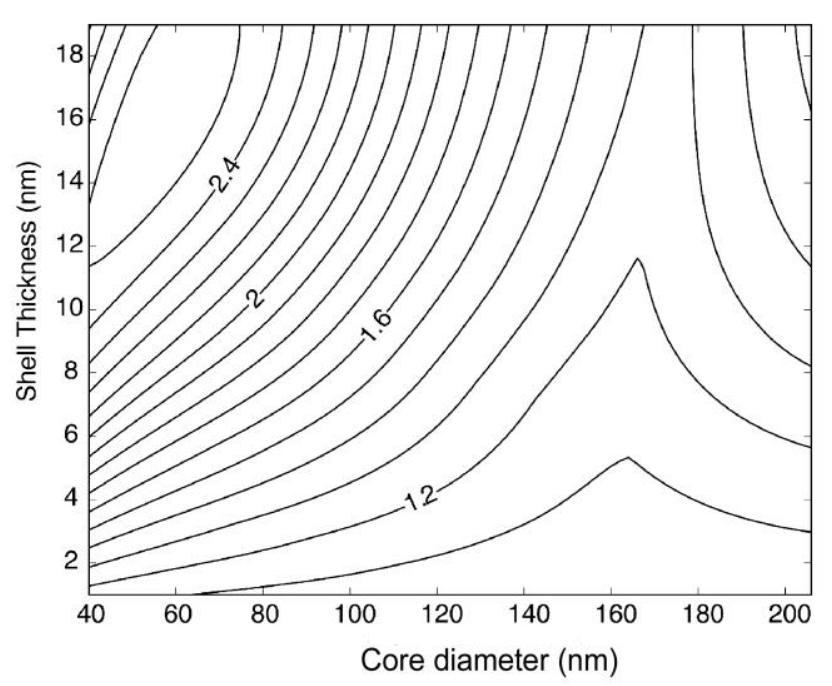

(b)

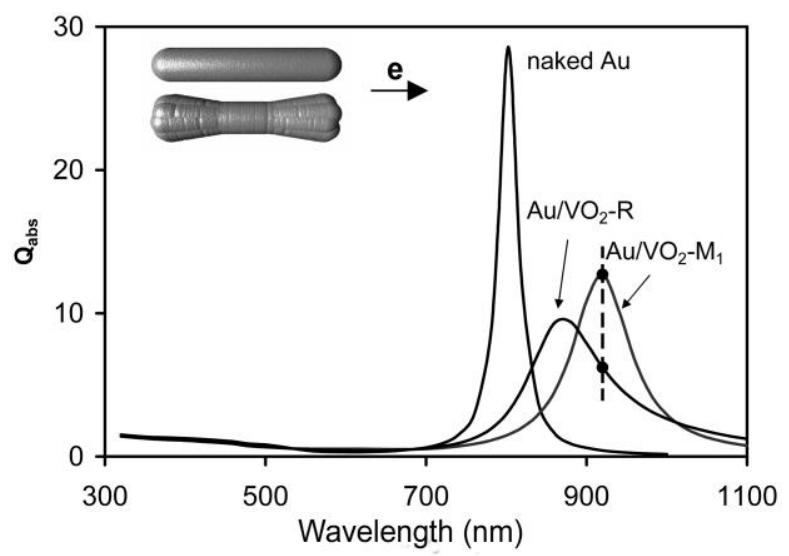

(d)

FIG. 1. Effect of the $\mathrm{VO}_{2}-\mathrm{M}_{1} \leftrightarrow \mathrm{VO}_{2}-\mathrm{R}$ phase transformation on the optical properties of composite $\mathrm{Au}+$ $\mathrm{VO}_{2}$ nanoparticles. (A) Experimental data of Maaza[16], redrawn with permission. The hatched region illustrates the shift in the position of peak plasmon resonance (B) Optimization of $\phi$ (out-of-paper axis on figure) for $\mathrm{Au} @ \mathrm{VO}_{2}$ particles. (C) Optimum $\mathrm{Au} @ \mathrm{VO}_{2}$ core-shell particle with schematic of particle shown as inset. (D) Gold nanorods of $100 \mathrm{~nm}$ length with end caps of $\mathrm{VO}_{2}$, compared to naked gold rod, with schematic of particles shown as inset. A numerical model of 13,000 dipoles for the plain gold rod, and 20,000 dipoles for the coated rod, was used in the calculation.

However, a solid gold nanosphere has a limited color gamut, whereas that of gold nanoshells or nanorods is more extensive and more sensitive to small variations in dielectric properties[14]. In Figure 2a we show how $\phi$ varies as a function of wavelength for $\mathrm{VO}_{2}$ nanospheres of $120 \mathrm{~nm}$ diameter, and varying thickness of Au shell. It is evident that the difference in optical properties between low 
temperature and high temperature particles increases as the wavelength increases, and that values of $\phi$ of over 12 can be achieved. The effect can be explored over a range of core radii and shell thicknesses (Figure 2b).

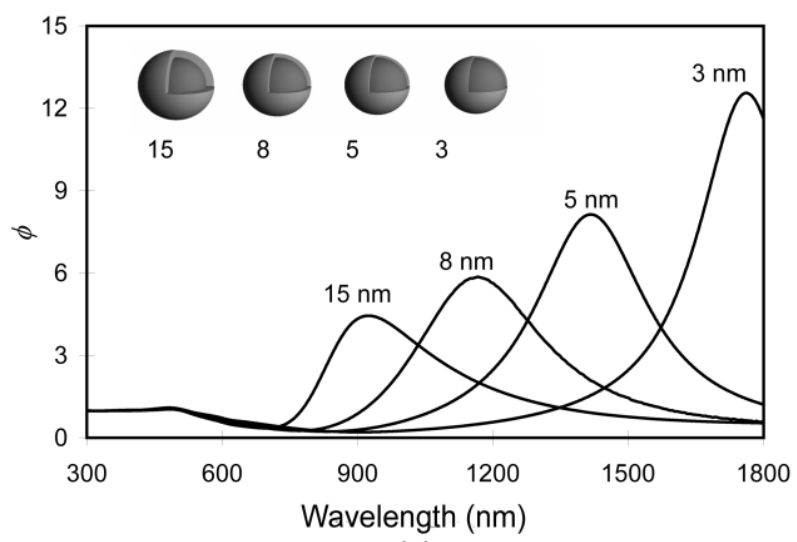

(a)

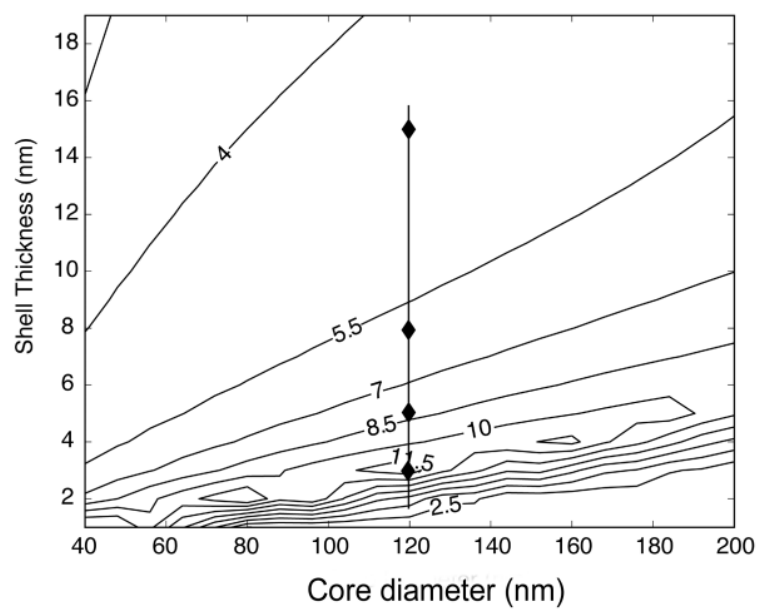

(b)

FIG. 2. Effect of the $\mathrm{VO}_{2}-\mathrm{M}_{1} \leftrightarrow \mathrm{VO}_{2}-\mathrm{R}$ phase transformation on the optical properties of $\mathrm{VO}_{2} @ \mathrm{Au}$ particles. (A) Variation of $\phi$ for a $\mathrm{VO}_{2}$ core of $120 \mathrm{~nm}$ diameter and various thicknesses of $\mathrm{Au}$ shell. (B) Plot of $\phi_{\max }$ for a wide range of core and shell dimensions, with the positions of the particles of (A) indicated.

In the case of photothermal therapeutic treatments based on plasmonic heating, laser irradiation of unhealthy tissues is applied in order to selectively destroy them while avoiding collateral damage to healthy cells. Gold nanospheres, shells or rods are concentrated in the target cells by active or passive means[23], following which a laser with a frequency matching the plasmon resonance of the nanoparticles is used to irradiate the tissue. The optimum transparency through human skin is between 
800 and $1000 \mathrm{~nm}[24]$. Therefore, to optimize the selective heating effect, the plasmon resonance of the nanoparticle should also be tuned to fall in this range. An example of a suitable particle and its associated optical absorption spectrum is shown in Figure $3 \mathrm{a}$.

The temperature and surface heat flux of a plasmonically-active nanoparticle under laser irradiation has been the subject of several investigations[6, 25-29], and we note that the assumption of adiabatic heating of the particle is only reasonable if $\mathrm{t}<<t_{\mathrm{T}}$, where $t_{\mathrm{T}}$, the characteristic time between particle and medium[27] provides an indication of whether transient or steady state phenomena are dominant, and is

$$
t_{T} \approx \frac{a_{0}^{2}}{4 \chi_{m}}
$$

where $a_{0}$ is the particle radius and $\chi_{m}$ is the thermal diffusivity of the medium which we take here as $1.53 \times 10^{-7} \mathrm{~m}^{2} / \mathrm{s}$, the value for liquid water. The applicable $t_{\mathrm{T}}$ for the model system shown in Figure $3 \mathrm{a}$ is $\sim 6 \mathrm{~ns}$, indicating that heat transfer out of these tiny particles reaches a quasi-steady state in about that time.

Here we will invoke the Pustovalov[27] model for transient heat generation and dissipation in a sphere immersed in an infinite conductor at temperature $T_{\infty}$ to simulate the behavior of the $\mathrm{VO}_{2}$ core-Au shell identified in Figure 3a. This solution is applicable only for time $t>t_{\mathrm{T}}$. Like others [26, 27] we will neglect heat transfer out of the particle by radiation. Although it is reasonable to use a conductive model for short times $(t<<1 \mathrm{~s})$ it should be noted that this does overestimate heat transfer rates out of the particle[25], the error becoming significant as $t$ approaches $1 \mathrm{~s}$ and convection becomes important[6]. The error is however negligible for the high speed transient phenomena considered here. With these caveats in mind, the temperature $T_{\mathrm{p}}$ in a particle subject to a laser flux $I_{\mathrm{o}}$ while immersed in an infinite medium is[27]

$$
T_{p}=T_{\infty}+\frac{I_{0} Q_{a b s} a_{0}}{4 k_{m}}\left[1-e^{-B t}\right]
$$

where $k_{\mathrm{m}}$ is the thermal conductivity of the medium, here water at $0.611 \mathrm{~W} / \mathrm{m} / \mathrm{K} . B$ is given by

$$
B=\frac{3 k_{m}}{c_{p} \rho_{p} a_{0}^{2}}
$$

where $c_{\mathrm{p}}$ is the particle's heat capacity (taken here to be the mass-weighted average of $\mathrm{Au}$ and $\mathrm{VO}_{2}$ which is $\sim 250 \mathrm{~J} / \mathrm{kg} / \mathrm{K}$ for this particular particle) and $\rho_{\mathrm{p}}$ is the average density of the particle $(11,640$ 
$\mathrm{kg} / \mathrm{m}^{3}$ in this specific case). Note that, in this geometry and provided $t>>t_{\mathrm{T}}, T_{\mathrm{p}}$ is effectively independent of $t$ and hence of laser fluence, although not of laser intensity.

Although the particle shown in Figure $3 \mathrm{a}$ has an optimum $Q_{\mathrm{abs}}$ in the insulating condition at $\lambda=837 \mathrm{~nm}$, the optimum $\phi$ for the particle is at $\lambda=924 \mathrm{~nm}$. At this wavelength $Q_{\mathrm{abs}}$ for this particle with a $\mathrm{VO}_{2}-\mathrm{M}_{1}$ core is 1.13 , while for the $\mathrm{VO}_{2}-\mathrm{R}$ core it is 0.25 . We will assume continuous wave illumination for simplicity. If illuminated by a sufficiently powerful source at $924 \mathrm{~nm}$, the temperature of the particle at first rises, Figure 3b. The details of the dynamic process that unfolds are influenced by magnitude of the hysteresis in phase transition temperatures which varies from as little as $1 \mathrm{~K}$ to as much as $50 \mathrm{~K}$ depending on the structure and morphology of the $\mathrm{VO}_{2}[17,30,31]$. Here we will examine two scenarios, a $5 \mathrm{~K}$ hysteresis and a $50 \mathrm{~K}$ hysteresis. In the first case the up-transformation (to $\mathrm{VO}_{2}-\mathrm{R}$ ) occurs at $340 \mathrm{~K}$ and the down transformation (to $\mathrm{VO}_{2}-\mathrm{M}_{1}$ ) at $335 \mathrm{~K}$, while in the second the corresponding temperatures are taken to be 300 and $350 \mathrm{~K}$.

Considering the first scenario, at $340 \mathrm{~K}$ the transformation of the core to $\mathrm{VO}_{2}-\mathrm{R}$ occurs, which reduces $Q_{\text {abs }}$ of the composite particle to only 0.25 , preventing further increase (dashed line) of the temperature of the particle taking place. The available evidence indicates that this phase transition will be rapid, taking place within 1 ps $[32,33]$. Heat generation in the particle with the $\mathrm{VO}_{2}-\mathrm{R}$ core is now significantly reduced, and the temperature of the particle will attempt to relax, in a few nanoseconds[25] (taken here for the purpose of our argument to be $\sim 2 \mathrm{~ns}$ ) to the appropriate equilibrium temperature for $\mathrm{VO}_{2}-\mathrm{R}$. However, once the temperature has fallen to $\sim 335 \mathrm{~K}$ transformation to $\mathrm{VO}_{2}-\mathrm{M}_{1}$ occurs, the particle heats up, and the cycle would repeat, ad infinitum. It is evident, Figure $3 c$ however that there is a limiting upper laser intensity $\left(4.56 \times 10^{9} \mathrm{~W} / \mathrm{m}^{2}\right.$ for the present example) at which even the steady state temperature of a particle with a $\mathrm{VO}_{2}-\mathrm{R}$ core is already $335 \mathrm{~K}$. Such a particle can no longer transform back to the $\mathrm{VO}_{2}-\mathrm{M}_{1}$ phase. Similarly, no self-regulation is possible if the laser intensity is insufficient to even raise the temperature of a particle with a $\mathrm{VO}_{2}-\mathrm{M}_{1}$ core above the phase transition temperature of $340 \mathrm{~K}$, Figure $3 \mathrm{~d}$, providing a lower limit $\left(1.15 \times 10^{9} \mathrm{~W} / \mathrm{m}^{2}\right.$ in the present example). The situation in the event of a $50 \mathrm{~K}$ hysteresis represents a further limiting case, since the transformation temperature for $\mathrm{VO}_{2}-\mathrm{R} \rightarrow \mathrm{VO}_{2}-\mathrm{M}_{1}$ and that of the medium would now be the same $(300 \mathrm{~K})$. In this case no regulatronic behavior is possible unless the medium temperature is lowered.

In this example we deliberately selected a particle where the effect would occur at $\sim 900 \mathrm{~nm}$ since this could be of interest in photothermal therapeutic applications. However, the self-regulating effect would obviously operate over an even greater range of laser intensities if particles designed to operate deeper in the infra-red were used ( $c f$. Figures $2 \mathrm{a}$ and $2 \mathrm{~b}$ ). 

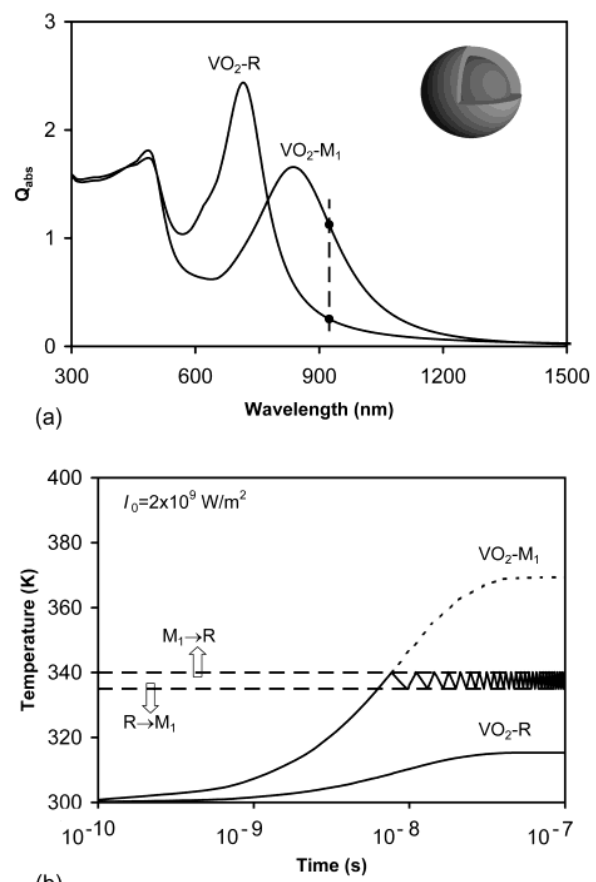

(b)
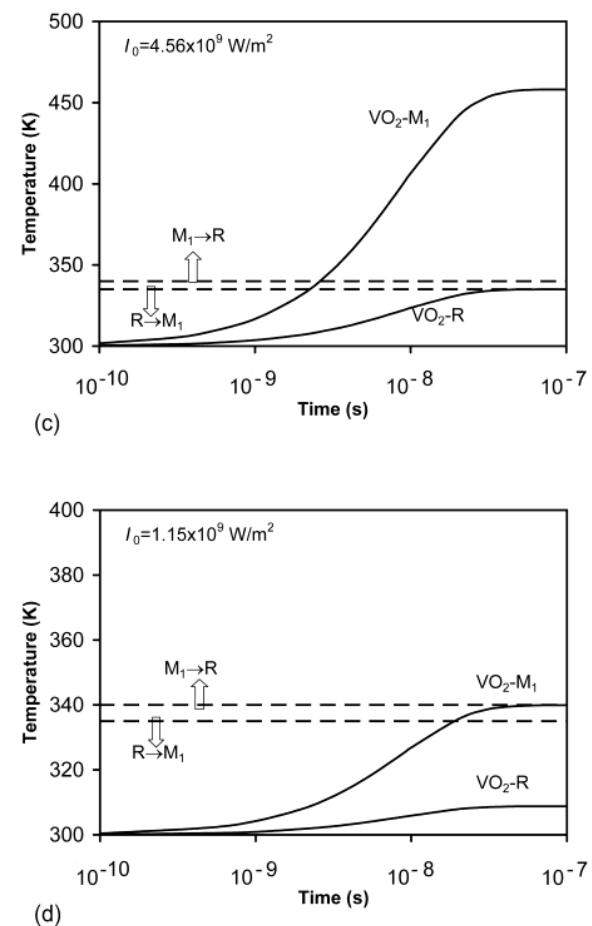

FIG. 3. Calculation of temperature in a $\mathrm{VO}_{2} @ \mathrm{Au}$ nanoparticle immersed in water and irradiated with a laser at various intensities. (A) Absorption efficiency of the particle with a $120 \mathrm{~nm}$ diameter core and a $15 \mathrm{~nm}$ thick shell which is optimized for service in the near-infrared, as a function of wavelength of light. (B) Predicted thermal behavior of particle of Figure 3A when illuminated with $924 \mathrm{~nm}$ light at $2 \times 10^{9} \mathrm{~W} / \mathrm{m}^{2}$ showing 'regulatron' behavior. (C) Predicted thermal behavior at upper limit of illumination intensity for 'regulatron' behavior. (D) Predicted thermal behavior at lower limit of illumination intensity for 'regulatron' behavior. 
At present, no way of producing $\mathrm{VO}_{2} @ \mathrm{Au}$ core-shell particles has been demonstrated. However, we have shown elsewhere that semishells, that is, partial nanoshells, can be engineered to have similar plasmonic properties to true nanoshells[34-36]. The attraction of semishells is that they can be produced by physical vapor deposition onto a suitable template. The present VO2@Au particles could conceivably be produced by evaporation of gold or silver onto nanospherical $\mathrm{VO}_{2}$ template particles. The temperature of the resulting regulatrons could be verified by one of several methods already disclosed in the literature[7, 37]\}.

In effect, these composite particles will behave as a 'smart' meta-materials, since they will manifest both intrinsic sensing and actuating functionalities. We define 'regulatrons' to be nano- or microparticles that can be engineered to maintain a constant temperature by means of a fast, self-adaptive and reversible transition in optical properties, even while being irradiated with a flux of varying intensity. Core-shell 'regulatrons' may be conceptually the simplest of these new structures, but we have shown that other nanoparticle geometries could also exhibit thermal regulation properties.

This work was supported by the Australian Research Council, and the University of Technology, Sydney. Computing resources were provided by the Australian Centre for Advanced Computing and Communication (ac3) in New South Wales.

[1] F. J. Morin, Physical Review Letters 3, 34 (1959).

[2] N. F. Mott, Metal Insulator Transitions (Taylor \& Francis, London, 1974).

[3] S. Biermann, A. Poteryaev, A. I. Lichtenstein, and A. Georges, Physical Review Letters 94, 026404 (2005).

[4] T. D. Manning and I. P. Parkin, Journal of Materials Chemistry 14, 2554 (2004).

[5] M. Soltani, M. Chaker, E. Haddad, and R. Kruzelesky, Measurement Science and Technology 17, 1052 (2006).

[6] N. Harris, M. J. Ford, and M. B. Cortie, J. Phys. Chem. B. 110, 10701 (2006).

[7] L. R. Hirsch, R. J. Stafford, J. A. Bankson, S. R. Sershen, B. Rivera, R. E. Price, J. D. Hazle, N. J. Halas, and J. L. West, Proceedings of the National Academy of Sciences 100, 13549 (2003).

[8] X. Huang, I. H. El-Sayed, W. Qian, and M. A. El-Sayed, Journal of American Chemistry Society 128, 2115 (2006).

[9] D. Pissuwan, S. Valenzuela, and M. B. Cortie, Trends Biotechnol. 24, 62 (2006). 
[10] C. F. Bohren and D. R. Huffman, Absorption and Scattering of Light by Small Particles (Wiley, New York, 1998).

[11] M. Cortie, X. Xu, H. Zareie, H. Chowdhury, and G. Smith, in Smart Materials, Nano-, and Micro-Smart Systems II, edited by S. F. Al-Sarawi (SPIE, Sydney, Australia, 2005), Vol. 5649, p. 565.

[12] D. P. O'Neal, L. R. Hirsch, N. J. Halas, J. D. Payne, and J. L. West, Cancer Letters 209, 171 (2004).

[13] A. O. Govorov, W. Zhang, T. Skeini, H. Richardson, J. Lee, and N. A. Kotov, Nanoscale Res Lett, DOI 10.1007/s11671 (2006).

[14] K. L. Kelly, E. Coronado, L. L. Zhao, and G. C. Schatz, J. Phys. Chem. B 107, 668 (2003).

[15] Y. Sun and Y. Xia, Analytical Chemistry 74, 5297 (2002).

[16] M. Maaza, O. Nemraoui, C. Sella, and A. C. Beye, Gold Bulletin 38, 100 (2005).

[17] G. Xu, Y. Chen, M. Tazawa, and P. Jin, J. Phys. Chem. B. 110, 2051 (2006).

[18] E. Cavanna, J. P. Segaud, and J. Livage, Mater. Res. Bull. 34, 167 (1999).

[19] J. Y. Suh, E. U. Donev, R. Lopez, L. C. Feldman, and R. F. Haglund, Applied Physics Letters 88, 133115 (2006).

[20] O. P. Mikheeva and A. I. Sidorov, Technical Physics 48, 602 (2003).

[21] B. T. Draine and P. J. Flatau, J. Opt. Soc. Am. A 11, 1491 (1994).

[22] H. W. Verleur, A. S. Barker, and C. N. Berglund, Physical Review 172, 788 (1968).

[23] S. E. McNeil, J. Leukoc. Biol. 78, 585-594 (2005).

[24] C. R. Simpson, M. Kohl, M. Essenpreis, and M. Copey, Phys. Med. Biol. 43, 2465-2478 (1998).

[25] M. Hu and G. V. Hartland, J. Phys. Chem. B 106, 7029 (2002).

[26] C. M. Pitsillides, E. K. Joe, X. Wei, R. R. Anderson, and C. P. Lin, Biophysical Journal 84, 4023 (2003).

[27] V. K. Pustovalov, Chemical Physics 308, 103-108 (2005).

[28] S. Link, C. Burda, B. Nikoobakht, and M. A. El-Sayed, Journal of Physical Chemistry B 104, $6152(2000)$.

[29] V. P. Zharov, K. E. Mercer, E. N. Galitovskaya, and M. S. Smeltzer, Biophys. J. 90,619 (2006).

[30] R. Lopez, T. E. Haynes, L. A. Boatner, L. C. Feldman, and R. F. Haglund, Physical Review B 65, 224113 (2002).

[31] H. S. Choi, J. S. Ahn, J. H. Jung, T. W. Noh, and D. H. Kim, Physical Review B 54, 4621 (1996).

[32] A. Cavalleri, C. Tóth, C. W. Siders, J. A. Squier, F. Ráksi, P. Forget, and J. C. Kieffer, Physical Review Letters 87, 237401 (2001). 
[33] R. Lopez, R. F. Haglund, L. C. Feldman, L. A. Boatner, and T. E. Haynes, Applied Physics Letters 85, 5191 (2004).

[34] J. Liu, B. Cankurtaran, G. McCredie, M. Ford, L. Wieczorek, and M. Cortie, Nanotechnology 16, $3023(2005)$.

[35] J. Liu, B. Cankurtaran, L. Wieczorek, M. J. Ford, and M. B. Cortie, Advanced Functional Materials, in press (2006).

[36] J. Liu, A. I. Maaroof, L. Wieczorek, and M. B. Cortie, Advanced Materials 17, 1276 (2005).

[37] V. Zharov, Optics Letters 28, 1314 (2003). 